\title{
2 ADVANCES IN EUROPEAN SEA BASS GENOMICS AND FUTURE
}

\section{PERSPECTIVES}

4 Bruno Louro ${ }^{1}$, Deborah M. Power ${ }^{1}$, Adelino V.M. Canario ${ }^{15}$

5

$6{ }^{1}$ CCMAR- Centre of Marine Sciences, University of Algarve, Campus de Gambelas, 8005-139 Faro, Portugal

7 \$corresponding author; email: acanario@ualg.pt, phone: +351-289-800925

8 


\section{Abstract}

11 Only recently available sequenced and annotated teleost fish genomes were restricted to a few model 12 species, none of which were for aquaculture. Application of Marker Assisted Selection for improved 13 production traits had been largely restricted to the salmon industry and genetic and Quantitative Trait 14 Loci (QTL) maps were available for only a few species. With the advent of Next Generation 15 Sequencing the landscape is rapidly changing and today the genomes of several aquaculture species 16 have been sequenced. The European sea bass, Dicentrarchus labrax, is a good example of a 17 commercially important aquaculture species in Europe for which in the last decade a wealth of 18 genomic resources, including a chromosomal scale genome assembly, physical and linkage maps as 19 well as relevant QTL have been generated. The current challenge is to stimulate the uptake of the 20 resources by the industry so that the full potential of this scientific endeavour can be exploited and 21 produce benefits for producers and the public alike.

\section{Key words}

24 Genomics; Genetics; Aquaculture; Selective breeding program; European sea bass. 
27 As little as a decade ago the only available fish genomes were from model fish species: Fugu rubripes [1], Tetraodon nigroviridis [2], Danio rerio [3], Oryzias latipes [4] and Gasterosteus aculeatus [5].With the "next generation sequencing" revolution, the flood of genomic and genetic data has grown exponentially and recently several genetics and genomics resources, including transcriptomes and genomes of economically relevant fish species have been published, e.g. [6-9] and [10] for a review. Despite these advances, so far the impact on aquaculture of new technologies in genome analysis coupled to a parsimonious breeding program is still limited [11]. This is particularly true in the Mediterranean area where intensive models of production have only recently to newly adopted aquaculture species of interest for which available resources may be limited.

\section{European sea bass aquaculture history and genetics resources}

The European sea bass is a gonochoristic marine teleost fish, distributed in temperate European coastal areas of the Northeast Atlantic Ocean and Mediterranean Sea. Its intensive exploitation as an aquaculture species is relatively recent and production is concentrated predominantly in the Mediterranean basin. It was initially cultivated in semi-intensive lagoon systems but since the 1980's production has become progressively more intensive due to its high commercial value. Total production of European sea bass was 126 thousand tonnes in 2010, with a market value of 500 million Euro [12]. The expansion of European sea bass aquaculture production throughout Europe and the associated increase in its commercial importance has been the catalyst that has led in a 
relatively short space of time to a significant body of scientific and technical knowledge about this species. The bulk of the research carried out on the European sea bass has largely occurred over the past twenty years and encompasses basic biology through to modern day genetics and genomics.

The European sea bass has in the last 10 years moved to the forefront of aquaculture species in terms of availability of genetic and genomic resources. The production of genomics and genetics tools for this species has been a community wide effort that has involved numerous scientists in Europe and in a large part has been driven by European Commission funded consortia. Outputs from such European projects include high density linkage and synteny maps, a radiation hybrid map, transcriptome data [13-20], a high quality draft genome sequence (NCBI bioproject accession: PRJEA39865) [19, 21, 22] and mapped QTLs for economic traits [15, 16, 23-25]. Table 1 lists publicly available genetic, genomic and/or transcriptomics resources for European sea bass and sustainable breeding program for European sea bass analogous to what has been developed for terrestrial farm animal production [26, 27].

\section{Genetics \& Genomics trends in research \& industry}

Selective breeding in aquaculture is mostly done by mass selection of the previous generation, or through family based selection. While mass selection is based only on selected parentage phenotypic values to identify the best individuals (selection candidates) in terms of their genetic potential for the desired traits, within family selection is based on breeding values (calculated through phenotype measurements and pedigree information) of the fish that is the target of selection and incorporating information on its relatives $[28,29]$. Selection based on genomic information is still a novelty in aquaculture, and there are relatively few examples of marker assisted selection (MAS) [30, 31]. One example of successful application of MAS is in salmonids, in which a major quantitative trait locus 
73 (QTL) affecting resistance to infectious pancreatic necrosis was selected by incorporating marker

74 information in the selective breeding program [32].

75 Alternative or complimentary approaches and strategies are required to MAS, which despite its 76 utility has inherent weaknesses linked to the limited number of QTL flanking markers used which 77 means only a fraction of the total genetic variance is captured [33]. An alternative approach to tracing a limited number of QTLs with markers is to trace all the QTL genome wide. This can be done by dividing the entire genome into chromosome segments, by adjacent markers with such 80 density that the population-wide linkage disequilibrium between markers and QTL is utilised to 81 generate the predicted genetic merit of the individual. This method has been termed genomic selection (GS) [34], but needs a dense set of markers across the genome. Thus, genomic selection integrated with next-generation-sequencing (NGS) promises to be of great potential to create genomic information of added value for the accuracy of genomic prediction and genome wide associations studies (e.g. finding causal mutations). The GS approach can potentially be done either by genotyping with Restriction site Associated DNA (RAD) [35], Genotyping-by-Sequencing (GBS) [36], or by whole genome re-sequencing [37] methodologies as illustrated by the 1000 bull genomes project (http://www.1000bullgenomes.com/). Simulations based upon standard aquaculture breeding practices of the gains (improved growth, disease resistance, etc.) suggest that genome-wide selection will result in high genetic gain for a typical family [38].

\subsection{Genomic selection approach}

92 GS can be seen as a new form of scale-up MAS with genetic markers densely covering the whole 93 genome identifying the full suite of QTLs of a given trait genome-wide. With the ease of production 94 of large single nucleotide polymorphism (SNP) markers data and lower the genotyping costs, the 95 limitation today may be in the initial steps, namely of obtaining a reference population with robust 96 phenotype data (and posterior prediction tuning) for the genomic prediction of phenotypes and 
97 breeding values with higher accuracies and better control of inbreeding [39]. To calculate the genomic estimated breeding value (gEBV), a reference population is genotyped and phenotyped in order to obtain a prediction equation which basically is the sum of the substitution effects over all SNPs. Selection candidates can then be screened through genotyping to choose the breeders by and obtain predictions of the phenotypes [34]. This approach is particularly useful in aquaculture species that reproduce by mass spawning, as it eliminates the steps of pedigree recording because all pedigree information and inbreeding control can be inferred from the SNP data [40]. The viability of applying GS in aquaculture will come from a balance between the cost of dense genotyping and the added gains the approach delivers compared to traditional mass or family-based selection. The cost of genotyping will depend on the cost per individual and how many individuals have to be genotyped, both initially in the test population to obtain marker estimates, and then on the number of candidates selected based on estimated breeding values.

\subsection{Transcriptomic approaches}

110 NGS is not only revolutionizing genetics by changing the scale and density of genotyping genome wide, it is also facilitating the identification of QTL through genome expression studies identify underlying genes and their respective levels of expression in order to understand the genetic pathways that underlie the traits effects. The European sea bass whole genome assembly [21] is an important tool for re-sequencing or gene expression studies. For example, SuperSAGE (Serial Analysis Gene Expression) combined with next generation sequencing was found to be an effective means of single nucleotide variant (SNV) calling [41] (Figure 1). SuperSAGE tags, obtained in the context of a study to analyse growth rates, were mapped to the draft genome sequence of the European sea bass and resulted in the identification of 506 SNVs and 257 one base indels that were directly linked to genes, mostly in the 3' UTR of the gene region. From the 763 candidate markers it was possible to obtain the identity of the genes for about half (387) of the identified polymorphisms. 
122 SAGE library (brain, liver, white muscle), that represented 47.071 unique transcripts were identified.

123 SuperSAGE or digital gene expression (DGE) potentially has more quantification depth than 124 RNAseq (mRNA) for the same amount of edited reads output from a sequence run, since in 125 SuperSAGE one tag corresponds to one transcript molecule count while with RNAseq one transcript 126 molecule can generate several reads. This is set from library construction, where SuperSAGE reads 127 are always originated from a single position loci (normally endonuclease EcoP15I restriction site) in 128 the a cDNA [42], while in RNAseq the reads are randomly originated at any position of the mRNA. 129 This superSAGE data was obtained by SOLID4 sequencing technology. For SNV calling, the most 130 relevant characteristic of SOLID sequencing is the double encoded nature of colour space sequences. 131 This together with fact that read quality could be confirmed by the presence of the adaptor A 132 sequence at the end of the unedited tag, increasing considerably the confidence regarding SNV false 133 positive calling. If there was any sequence error within the tag there would be a sequence misframe 134 and the end adaptorA would not be present.If it had been possible to use RNA samples from 135 individuals instead of pools of individuals it would have been possible to genotype individuals with 136 thousands of SNV at the same time obtain the gene expression quantification to be used as trait per 137 si. This would discriminate genes allele-specific expression (ASE) and another very interesting 138 possibility would be to apply genetical-genomics [43] in a single step using the gene expression 139 values as the trait and the genotypes, instead of methodologies in which gene expression is measured 140 and genotyping performed separately. RNA-seq can be used even to greater advantage for such 141 purpose of all in one gene expression Quantitative Trait Locus (eQTL) studies [44]. RNA-seq delivers ASE data, unlike microarrays, and also RNA-isoform expression discrimination [45].

\section{4. Conclusion}

144 European sea bass is now a member of the restrict club of aquaculture species rich in genomic and genetic resources, which until recently only included salmonids [46, 47]. The molecular tools and approach can be applied in selective breeding programs, especially in the case where traits of interest 
cannot be or are difficult to measure directly in the selection candidate fish, as for example disease

148 resistance or fillet quality. Not only can they be applied to improve selection accuracy but they can 149 also be used to manage, and control on-going breeding programs, such as to monitor the 150 maintenance of suitable levels of additive genetic variation within the broodstocks so that selection 151 for rapid genetic gain does not lead to decreased heterozygosity and increased inbreeding [48].

152 While until recently the lack of molecular genomic tools has hindered progress for the 153 implementation of a successful high yield selective breeding program, the limitations have now 154 shifted onto structural and economic issues related to the lack of appropriate facilities and 155 infrastructures to maintain established genetic groups, phenotyping methodologies and trained 156 personnel [46]. In salmon aquaculture the transfer of knowledge from research to industry is a long 157 term achievement that took years of investment of money and research and the establishment of a 158 strong collaboration between industry and $\mathrm{R} \& \mathrm{D}[11,29]$. The salmon story is decades long and is 159 one of success with the genetic gain achieved being estimated at approximately $14 \%$ per generation 160 with a global benefit/cost ratio estimated at 15/1 [11]. The current challenge is to stimulate the uptake of the resources by the European sea bass industry so that the full potential of this scientific endeavour can be exploited and produce benefits for producers and the public alike.

165 The research reported has been supported by the European Regional Development Fund (ERDF) through the COMPETE - Operational Competitiveness Programme and national funds through the Portuguese Foundation for Science and Technology (FCT) under the project "PEstC/MAR/LA0015/2011. BL benefited from a FCT postdoctoral fellowship (SFRH/BPD/89899/2012). 
172 1. Aparicio, S., Chapman, J., Stupka, E., Putnam, N., Chia, J.-m., Dehal, P., Christoffels, A., Rash,

S., Hoon, S., Smit, A., et al. (2002). Whole-genome shotgun assembly and analysis of the genome of Fugu rubripes. Science 297, 1301-1310.

2. Jaillon, O., Aury, J.-M., Brunet, F., Petit, J.-L., Stange-Thomann, N., Mauceli, E., Bouneau, L., Fischer, C., Ozouf-Costaz, C., Bernot, A., et al. (2004). Genome duplication in the teleost fish Tetraodon nigroviridis reveals the early vertebrate proto-karyotype. Nature 431, 946-957.

3. Howe, K., Clark, M.D., Torroja, C.F., Torrance, J., Berthelot, C., Muffato, M., Collins, J.E., Humphray, S., McLaren, K., Matthews, L., et al. (2013). The zebrafish reference genome sequence and its relationship to the human genome. Nature 496, 498-503.

4. Kasahara, M., Naruse, K., Sasaki, S., Nakatani, Y., Qu, W., Ahsan, B., Yamada, T., Nagayasu, Y., Doi, K., Kasai, Y., et al. (2007). The medaka draft genome and insights into vertebrate genome evolution. Nature 447, 714-719.

5. Jones, F.C., Grabherr, M.G., Chan, Y.F., Russell, P., Mauceli, E., Johnson, J., Swofford, R., Pirun, M., Zody, M.C., White, S., et al. (2012). The genomic basis of adaptive evolution in threespine sticklebacks. Nature 484, 55-61.

6. Chen, S., Zhang, G., Shao, C., Huang, Q., Liu, G., Zhang, P., Song, W., An, N., Chalopin, D., Volff, J.-N., et al. (2014). Whole-genome sequence of a flatfish provides insights into ZW sex chromosome evolution and adaptation to a benthic lifestyle. Nat. Genet. 46, 253-260.

7. Star, B., Nederbragt, A.J., Jentoft, S., Grimholt, U., Malmstrom, M., Gregers, T.F., Rounge, T.B., Paulsen, J., Solbakken, M.H., Sharma, A., et al. (2011). The genome sequence of Atlantic cod reveals a unique immune system. Nature 477, 207-210. 
193 8. Guyon, R., Rakotomanga, M., Azzouzi, N., Coutanceau, J.P., Bonillo, C., D'Cotta, H., Pepey, E., Soler, L., Rodier-Goud, M., D'Hont, A., et al. (2012). A high-resolution map of the Nile tilapia genome: a resource for studying cichlids and other percomorphs. BMC Genomics 13, 222.

9. Wang, S., Furmanek, T., Kryvi, H., Krossoy, C., Totland, G., Grotmol, S., and Wargelius, A. (2014). Transcriptome sequencing of Atlantic salmon (Salmo salar L.) notochord prior to

10. Canario, A.V.M., Bargelloni, L., Volckaert, F., Houston, R.D., Massault, C., and Guiguen, Y. (2008). Genomics toolbox for farmed fish. Reviews in Fisheries Science 16, 3-15.

11. Gjedrem, T. (2010). The first family-based breeding program in aquaculture. Reviews in Aquaculture 2, 2-15.

12. FAO (2012). The state of the world fisheries and aquaculture, (Rome: Food and Agriculture Organization of the United Nations).

13. Chistiakov, D.A., Hellemans, B., Haley, C.S., Law, A.S., Tsigenopoulos, C.S., Kotoulas, G., Bertotto, D., Libertini, A., and Volckaert, F.A. (2005). A microsatellite linkage map of the European sea bass Dicentrarchus labrax L. Genetics 170, 1821-1826.

14. Chistiakov, D., Tsigenopoulos, C., Lagnel, J., Guo, Y., Hellemans, B., Haley, C., Volckaert, F., and Kotoulas, G. (2008). A combined AFLP and microsatellite linkage map and pilot comparative genomic analysis of European sea bass Dicentrarchus labrax L. Anim. Genet. 39, 623-634.

15. Massault, C., Hellemans, B., Louro, B., Batargias, C., Van Houdt, J.K.J., Canario, A., Volckaert, F.A.M., Bovenhuis, H., Haley, C., and De Koning, D.J. (2010). QTL for body weight, morphometric traits and stress response in European sea bass Dicentrarchus labrax. Anim. Genet. 41, 337-345. 
16. Volckaert, F., Hellemans, B., Batargias, C., Louro, B., Massault, C., Van Houdt, J., Haley, C., de Koning, D.-J., and Canario, A. (2012). Heritability of cortisol response to confinement stress in European sea bass dicentrarchus labrax. Genetics Selection Evolution 44, 15.

17. Guyon, R., Senger, F., Rakotomanga, M., Sadequi, N., Volckaert, F.A.M., Hitte, C., and Galibert, F. (2010). A radiation hybrid map of the European sea bass (Dicentrarchus labrax) based on 1581 markers: Synteny analysis with model fish genomes. Genomics 96, 228-238.

18. Louro, B., Passos, A.L.S., Souche, E.L., Tsigenopoulos, C., Beck, A., Lagnel, J., Bonhomme, F., Cancela, L., Cerdà, J., Clark, M.S., et al. (2010). Gilthead sea bream (Sparus auratus) and European sea bass (Dicentrarchus labrax) expressed sequence tags: Characterization, tissuespecific expression and gene markers. Marine Genomics 3, 179-191.

19. Kuhl, H., Beck, A., Wozniak, G., Canario, A., Volckaert, F., and Reinhardt, R. (2010). The European sea bass Dicentrarchus labrax genome puzzle: comparative BAC-mapping and low coverage shotgun sequencing. BMC Genomics 11, 68.

20. Kuhl, H., Tine, M., Hecht, J., Knaust, F., and Reinhardt, R. (2011). Analysis of single nucleotide polymorphisms in three chromosomes of European sea bass Dicentrarchus labrax. Comparative Biochemistry and Physiology Part D: Genomics and Proteomics 6, 7075.

21. Tine, M., Kuhl, H., Gagnaire, P.-A., Louro, B., Desmarais, E., Martins, R.S.T., Hecht, J., Knaust, F., Belkhir, K., Klages, S., et al. (2014). The genome of European sea bass (Dicentrarchus labrax) provides insights into euryhalinity adaptation and Atlantic-Mediterranean divergence mechanisms. Nat Commun, submitted.

22. Kuhl, H., Tine, M., Beck, A., Timmermann, B., Kodira, C., and Reinhardt, R. (2011). Directed sequencing and annotation of three Dicentrarchus labrax L. chromosomes by applying 
Sanger- and pyrosequencing technologies on pooled DNA of comparatively mapped BAC clones. Genomics 98, 202-212.

23. Chatziplis, D., Batargias, C., Tsigenopoulos, C.S., Magoulas, A., Kollias, S., Kotoulas, G.,

(Dicentrarchus labrax L.) using a marker-based pedigree. Aquaculture 275, 81-87.

25. Saillant, E., Dupont-Nivet, M., Haffray, P., and Chatain, B. (2006). Estimates of heritability and genotype-environment interactions for body weight in sea bass (Dicentrarchus labrax L.) raised under communal rearing conditions. Aquaculture 254, 139-147.

26. de Koning, D.-J., Archibald, A., and Haley, C.S. (2007). Livestock genomics: bridging the gap between mice and men. Trends Biotechnol. 25, 483-489.

27. Sellner, E.M., Kim, J.W., McClure, M.C., Taylor, K.H., Schnabel, R.D., and Taylor, J.F. (2007). Applications of genomic information in livestock. J Anim Sci 85, 3148-3158.

28. Falconer, D., and Mackay, T. (1996). Introduction to quantitative genetics, 4th Edition, (Harlow, UK: Longman).

29. Gjedrem, T. ed. (2005). Selection and breeding programs in aquaculture (Dordrecht; New York: Springer).

30. Fernando, R., and Grossman, M. (1989). Marker assisted selection using best linear unbiased prediction. Genetics Selection Evolution 21, 467 - 477.

31. Sonesson, A.K. (2007). Marker-assisted selection in aquaculture breeding programs. Aquaculture 272, Supplement 1, S311. 
32. Houston, R.D., Haley, C.S., Hamilton, A., Guy, D.R., Tinch, A.E., Taggart, J.B., McAndrew, B.J., and Bishop, S.C. (2008). Major quantitative trait loci affect resistance to infectious pancreatic necrosis in Atlantic salmon (Salmo salar). Genetics 178, 1109-1115.

33. Dekkers, J.C.M. (2004). Commercial application of marker- and gene-assisted selection in livestock: Strategies and lessons. J Anim Sci 82, E313-E328.

34. Meuwissen, T.H.E., Hayes, B.J., and Goddard, M.E. (2001). Prediction of total genetic value using genome-wide dense marker maps. Genetics 157, 1819-1829.

35. Miller, M.R., Dunham, J.P., Amores, A., Cresko, W.A., and Johnson, E.A. (2007). Rapid and cost-effective polymorphism identification and genotyping using restriction site associated DNA (RAD) markers. Genome Res. 17, 240-248.

36. Elshire, R.J., Glaubitz, J.C., Sun, Q., Poland, J.A., Kawamoto, K., Buckler, E.S., and Mitchell, S.E. (2011). A robust, simple genotyping-by-sequencing (GBS) approach for high diversity species. PLoS ONE 6, e19379.

37. Stratton, M. (2008). Genome resequencing and genetic variation. Nat. Biotechnol. 26, 65-66.

38. Sonesson, A., and Meuwissen, T. (2009). Testing strategies for genomic selection in aquaculture breeding programs. Genetics Selection Evolution 41, 1-9.

39. Daetwyler, H.D., Calus, M.P.L., Pong-Wong, R., de los Campos, G., and Hickey, J.M. (2013). Genomic prediction in animals and plants: Simulation of data, validation, reporting, and benchmarking. Genetics 193, 347-365.

40. Power, D.M., Louro, B., Houston, R., Anjos, L., and Cardoso, J.C.R. (2011). Genomicproteomic research in Sparidae and its application to genetic improvement. In Sparidae. (Wiley-Blackwell), pp. 359-381.

41. Li, H., Ruan, J., and Durbin, R. (2008). Mapping short DNA sequencing reads and calling variants using mapping quality scores. Genome Res. 18, 1851-1858. 
42. Matsumura, H., Urasaki, N., Yoshida, K., Krüger, D., Kahl, G., and Terauchi, R. (2012). SuperSAGE: Powerful Serial Analysis of Gene Expression. In RNA Abundance Analysis, Volume 883, H. Jin and W. Gassmann, eds. (Humana Press), pp. 1-17.

43. Jansen, R.C., and Nap, J.-P. (2001). Genetical genomics: the added value from segregation. Trends Genet. 17, 388-391.

44. Gregg, C., Zhang, J., Weissbourd, B., Luo, S., Schroth, G.P., Haig, D., and Dulac, C. (2010). High-resolution analysis of parent-of-origin allelic expression in the mouse brain. Science $329,643-648$.

45. Sun, W., and Hu, Y. (2013). eQTL mapping using RNA-seq data. Statistics in Biosciences 5, 198-219.

46. Fjalestad, K.T., Moen, T., and Gomez-Raya, L. (2003). Prospects for genetic technology in salmon breeding programmes. Aquaculture Research 34, 397-406.

47. Moen, T., Baranski, M., Sonesson, A., and Kjoglum, S. (2009). Confirmation and fine-mapping of a major QTL for resistance to infectious pancreatic necrosis in Atlantic salmon (Salmo salar): population-level associations between markers and trait. BMC Genomics 10, 368.

48. Muir, J. (2005). Managing to harvest? Perspectives on the potential of aquaculture. Philos T R Soc B 360, 191-218.

49. Magnanou, E., Klopp, C., Noirot, C., Besseau, L., and Falcón, J. (2014). Generation and characterization of the sea bass Dicentrarchus labrax brain and liver transcriptomes. Gene $544,56-66$.

50. Sarropoulou, E., Galindo-Villegas, J., García-Alcázar, A., Kasapidis, P., and Mulero, V. (2012). Characterization of European sea bass transcripts by RNA Seq after oral vaccine against $V$. anguillarum. Marine Biotechnology 14, 634-642. 
51. Ferraresso, S., Milan, M., Pellizzari, C., Vitulo, N., Reinhardt, R., Canario, A., Patarnello, T., and Bargelloni, L. (2010). Development of an oligo DNA microarray for the European sea bass and its application to expression profiling of jaw deformity. BMC Genomics 11, 354.

52. Geay, F., Ferraresso, S., Zambonino-Infante, J., Bargelloni, L., Quentel, C., Vandeputte, M., Kaushik, S., Cahu, C., and Mazurais, D. (2011). Effects of the total replacement of fish-based diet with plant-based diet on the hepatic transcriptome of two European sea bass (Dicentrarchus labrax) half-sibfamilies showing different growth rates with the plant-based diet. BMC Genomics 12, 522.

53. Darias, M.J., Zambonino-Infante, J.L., Hugot, K., Cahu, C.L., and Mazurais, D. (2008). Gene expression patterns during the larval development of European sea bass (Dicentrarchus Labrax) by microarray analysis. Marine Biotechnology 10, 416-428.

54. Souche, E.L., Hellemans, B., Van Houdt, J.K., and Volckaert, F.A. (2012). Characterisation and validation of single-nucleotide polymorphism markers in expressed sequence tags of European sea bass. Molecular Ecology Resources in press.

55. Homer, N., Merriman, B., and Nelson, S.F. (2009). BFAST: An alignment tool for large scale genome resequencing. PLOS ONE 4, e7767.

56. Li, H., Handsaker, B., Wysoker, A., Fennell, T., Ruan, J., Homer, N., Marth, G., Abecasis, G., Durbin, R., and Subgroup, G.P.D.P. (2009). The sequence alignment/map format and SAMtools. Bioinformatics 25, 2078-2079.

57. Goecks, J., Nekrutenko, A., Taylor, J., and Team, T.G. (2010). Galaxy: a comprehensive approach for supporting accessible, reproducible, and transparent computational research in the life sciences. Genome Biology 11, R86. 


\section{Figure legends}

336 Figure 1. SNV discovery and mapping with SuperSAGE. Solid4 colour-space 26bp SuperSAGE 337 reads where aligned with the European sea bass genome using bfast-0.6.4e [55]. A SAM mapping 338 file for aligned and matched tags was sorted, indexed and converted to BAM format using "faidx", 339 "sort", “index", and "view" scripts from SAMtools program [http://samtools.sourceforge.net; 56]. $340 \mathrm{SNV}$ and indels (only one nucleotide) were filtered from the genome tag alignments containing more 341 than 10 overlapping tag sequences. SAMtools scripts ("pileup" and "varFilter") were used to select 342 SNV and indel candidates over a threshold of 20 and 50 Phred-scaled likelihood (aka SNP quality) 343 respectively resulting in $506 \mathrm{SNVs}$ and 257 indel. Identified and retrieved polymorphisms were 344 annotated using their positional coordinate against European sea bass GFF3 genomic gene annotation 345 file. This was done with "Operate on Genomic Intervals" tool in the Galaxy server 346 [https://main.g2.bx.psu.edu; 57]. 


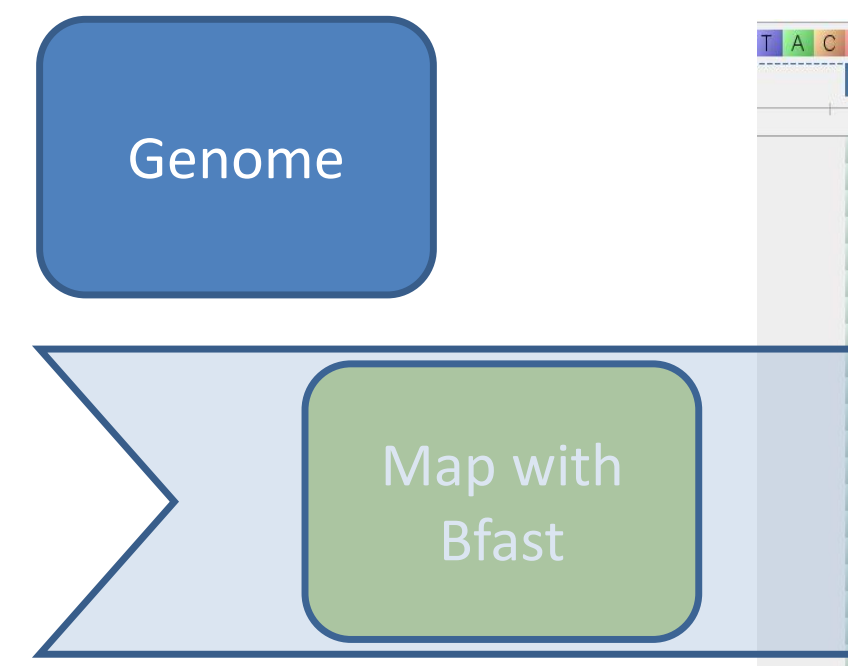

$A C G A C A C T A C T$ T CACA GC TGA G G A C A T G C T G

G A C A C T T A C T T T C A C A G C T G A G G A C A T G G A C A C T A G A C A C T A C T T C A A C A G C C T G A G G A C A T G $G A C C A C C T A A C T$ T C A C C A G C T T G A G G A C A T G $G A C C A C C T A A C D T$ T C A C A G G C T G A G G A C A T G $G A C A C C$ T A C T T C C A C C A G C C T G A G A A C A T G $G$ A C A C T A $A$ C T T T C A C C A G C C T G A G G A C A $A$ T G

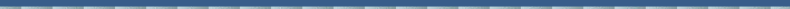
\begin{tabular}{lllllllllllllllllllllllllll}
$G$ & $A$ & $C$ & $A$ & $C$ & $T$ & $A$ & $C$ & $T$ & $T$ & $C$ & $A$ & $C$ & $A$ & $G$ & $C$ & $T$ & $G$ & $A$ & $G$ & $G$ & $A$ & $C$ & $A$ & $T$ & $G$ \\
$G$ & $A$ & $C$ & $A$ & $C$ & $T$ & $A$ & $C$ & $T$ & $T$ & $C$ & $A$ & $C$ & $A$ & $G$ & $C$ & $T$ & $G$ & $A$ & $G$ & $G$ & $A$ & $C$ & $A$ & $T$ & $G$ \\
\hline
\end{tabular} $G A C C A A C T$ A $C$ C T T T C A C C A G C C T G A G G A C A T G $G A C C A C C T A C C T$ T C A C A G C T G A G A A C A T G G A C C A C C T A A C C T T C C A C C A G G C T G G A G G G A C A T T G $G A C C A C C T A C C T$ T C A C A G G C T G A G A A C C A T G G A C C A C T TA A C T T T C A C A A G C T G A G G A C A T G $G$ A C A C C T A A C C T T T C A C C A G G C T T G A G G A A C C A T G G A C C A C C T A A C T T T C A C C A G G C T G A G G G A C A T G G A C C A C C T A C C T T C C A C A G G C T G A G A A C A T G $G A C C A C C$ T A C C T T T C A A C A A G C C T G G A G A A A C C A T G GA A A C A A T TCACAOCOTOAOACATO $G$ A C A C T A C T T C A C A G C T GA G G A C A T G G A C A C TA C T TCA C CA GC T G GA CA C TA C T T CA C CA OCT C OA G GACCA T G

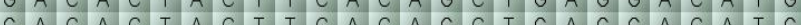

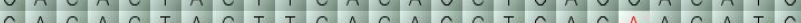
OACA C TA C T TCACAG C T GA G A A C A T G G A C A C T A C T T C A C CAOCOTGA G OACA T G $G A C A C C T A C C T T C A C A G C T G A G G A C A T G$ $G A C A C C T A C T T T C A C A G C T O A G G A C A T G$

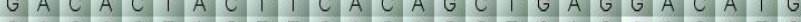
$\begin{array}{llllllllllllllllllllllllll}G & A & C & A & C & T & A & C & T & T & C & A & C & A & G & C & T & G & A & G & A & A & C & A & T & G \\ G & A & C & A & C & T & A & C & T & T & C & A & C & A & G & C & T & G & A & G & G & A & C & A & T & G\end{array}$ 375.1564

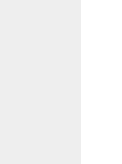

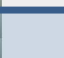
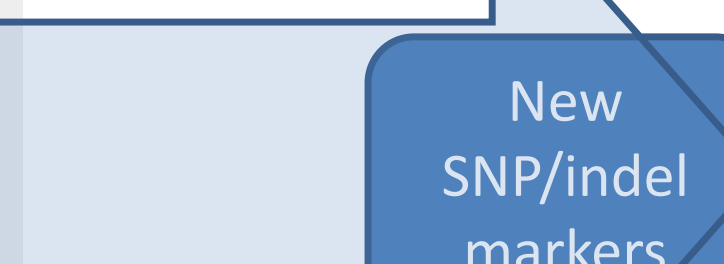
markers

\section{0 million} tags

SNP screen with SAMtools 
Table 1. Genetic, genomic and transcriptomics publicly available resources.

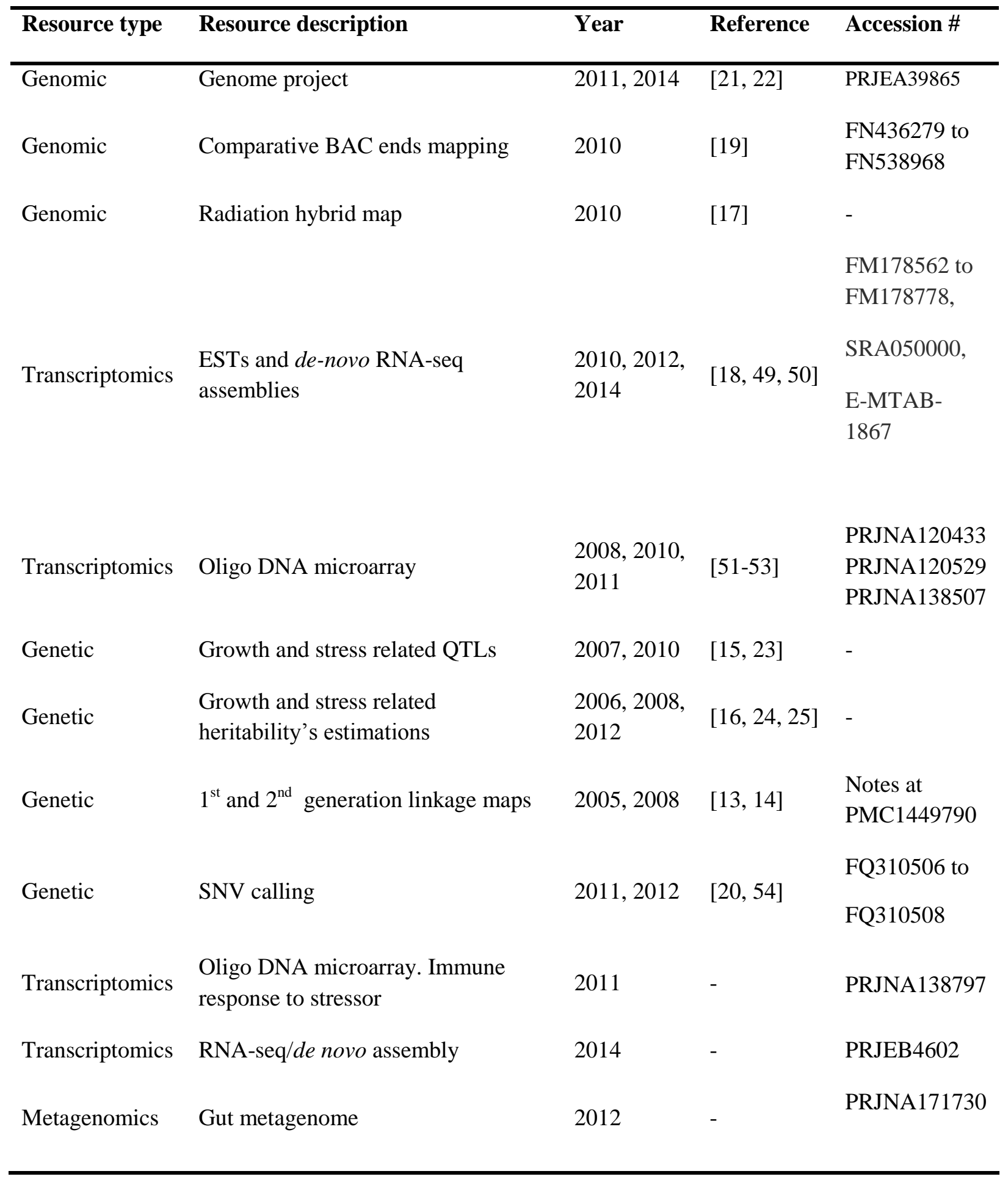

